\title{
Multimodality treatment in unresectable cholangiocarcinoma
}

\author{
Prof. Mahdi Aghili, MD', Prof. Hazhir Saberi, MD², Mahtab Mojtahed zadeh, MD³, Fatemeh Jafari, MD', \\ Mohammad-Mehdi Mehrabinejad, MD4, Habibollah Dashti, MD5, Pejman Saberi, MD6. Niloofar Ayoobi Yazdi, MD, \\ Assoc. Prof. 2 \\ IRadiation Oncology Research Center, Cancer Institute, Imam Khomeini Hospital, Tehran University of Medical Sciences, Tehran, Iran, \\ 2Department of Radiology, Liver Transplantation Research Center, Imam Khomeini Hospital, Tehran University of Medical Sciences, Tehran, \\ Iran, ${ }^{3}$ Radiology Department, Tehran University of Medical Sciences, Tehran, Iran, ${ }^{4}$ Department of Radiology, Advanced Diagnostic and \\ Interventional Radiology Research Center (ADIR), Tehran University of Medical Sciences, Tehran, Iran, ${ }^{5}$ Liver Transplantation Research Center, \\ Imam Khomeini Hospital, Tehran University of Medical Sciences, Tehran, Iran, ‘University of Debrecen, Faculty of Debrecen, Hungary
}

\begin{abstract}
Purpose: Cholangiocarcinomas (CCs) are rare and highly malignant cancers. Although there are different treatment protocols for treatment of cholangiocarcinoma, we aimed to investigate a survival rate of patients with unresectable extrahepatic CCs (ECCs) receiving multimodality therapeutic protocol (MTP) (biliary drainage + external beam radiotherapy [EBRT] + brachytherapy and systemic chemotherapy). Similarly, we aimed to identify a relationship between survival time and associated factors in treatment outcome.

Material and methods: This retrospective study was performed on patients with ECC, who were referred to our university hospital between 2012 and 2015, and their imaging were diagnosed as unresectable. Patients underwent MTP including internal-external drainage catheter (F10-12) with insertion under fluoroscopy guidance, EBRT with 25-28 fractions and concurrent chemotherapy using capecitabine (Xeloda) $825 \mathrm{mg} / \mathrm{m}^{2}$ at the days of radiotherapy, followed by brachytherapy (BT) with iridium-192 $\left({ }^{192} \mathrm{Ir}\right)$ or cobalt-60 $\left({ }^{60} \mathrm{Co}\right)$ sources for $21 \mathrm{~Gy}$ in 3 consecutive days. Demographic variables, complications, laboratory tests, imaging findings, and survival time (OS - overall survival after diagnosis; CS - survival after catheter placement) were recorded.

Results: A total of 38 patients, with mean SD age $=58.08(9.80)$ years, male $=22(57.9 \%)$, were evaluated. According to Bismuth-Corlette classification, $15(39.5 \%)$ were in stage IIIA, 5 (13.2\%) were in stage IIIB, $10(26.3 \%)$ were in stage IV, and $8(21.2 \%)$ were undefined. Of those, 21 (55.3\%), 15 (39.5\%), and $17(44.7 \%)$ were involved with liver parenchyma, great vessels, and regional lymph nodes, respectively. Mean SD of OS was 15.11 (8.10) months (median = 15; 95\% CI: 13.25-16.69), and CS was 2-29 months (mean SD = 11.71 (7.29); median $=10$; 95\% CI: 10.05-13.37). Further analysis revealed a considerable decrease in OS and CS in those with an involvement of liver parenchyma, great vessels, regional lymph nodes, and Bismuth type IV.

Conclusions: Multimodality therapeutic approach in patients with inoperable ECCs could definitely improve their survival time and decrease complications. Survival time is significantly depending on tumor staging, gender, and involvement of liver parenchyma, great vessels, and regional lymph nodes.

Key words: cholangiocarcinoma, drainage, radiotherapy, brachytherapy, chemotherapy, survival.

\section{Purpose}

Cholangiocarcinomas (CCs), including intrahepatic cholangiocarcinoma (ICC) and extrahepatic cholangiocarcinoma (ECC), are rare, devastating, and highly malignant cancers of bile duct, associated with high mortality due to its aggressive and refractory nature and delayed in diagnosis $[1,2]$. These tumors account for about $10-20 \%$ of hepatobiliary cancer-related deaths [3]. Median survival of patients with locally advanced and unresectable mass is approximately ten months [4], and five-year survival is less than $10 \%$ [5].

Clinical presentation of ECCs is non-specific, mostly occurring in the elderly population, and patients often present with no specific symptoms. However, some patients might present with rather early symptoms of biliary obstructions or late constitutional symptoms of abdominal mass or discomfort [3,6]. Diagnosis of ECCs requires a multidisciplinary approach including clinical laboratory tests, radiography, and endoscopic modalities [7]. CCs
Address for correspondence: Niloofar Ayoobi Yazdi, Assist. Prof. of Radiology, Department of Radiology, School of Medicine, Advanced Diagnostic and Interventional Radiology Research Center (ADIR), Imam Khomeini Hospital, Tehran University of Medical Sciences, 1419733141 Tehran, Iran, phone: +9821 66581535, +98913 356 0564, e-mail: nayoobi@sina.tums.ac.ir
Received: 15.12 .2019

Accepted: 18.02 .2020

Published: 30.04 .2020 
classification is based on anatomical location, macroscopic growth pattern, microscopic characteristics, and cellular origin [6]. Treatment of choice in CCs is based on patient's health status, biochemical features of tumor, surgical applicability, and stage of the disease [7]. Surgical resection of the tumor is the first-line therapy with long-term survival in good surgical candidates [8]; however, only half of the tumors at the time of surgical exploration are resectable [9]. The initial step in the palliative treatment of patients with unresectable tumors is percutaneous transhepatic biliary decompression, which reduces the symptoms of the patient, such as icterus [10]. Due to its refractory nature and local recurrence, combined therapy with radiotherapy and chemotherapy has been suggested [9]. Several treatment protocols using radiotherapy (intraluminal brachytherapy and external beam radiotherapy), combined with systemic chemotherapy and biliary decompression have been recommended in different studies, providing patients with higher survival time and better local control, using intraluminal brachytherapy to deliver a high-dose of radiation to the tumor for enhancement of local control as well as palliation [10].

The current study was designed to investigate the survival rate of patients with unresectable ECCs in patients receiving multimodality therapeutic protocol (MTP) (biliary drainage + external beam radiotherapy [EBRT] + brachytherapy and systemic chemotherapy). Similarly, we aimed to identify the relationship between survival time and associated factors in treatment outcome, including imaging characteristics of the tumor as well as demographic features and staging of the tumor.

\section{Material and methods}

\section{Study design and participants}

This study was conducted at our university hospital. All patients with hilar ECCs, in which the tumor was diagnosed as unresectable in imaging findings (magnetic resonance imaging [MRI], computed tomography [CT] scan, endoscopic ultrasonography), and based on an expert surgeon opinion, between 2012 and 2015, were included into the study. The decision of MTP was made by a multidisciplinary expert team consisting of a radiation oncologist, radiologist, and hepatobiliary surgeon.

Unresectability criteria for patients with ECC [11] included: 1. Medical comorbidities that restrict the patient's capability to undergo major surgery; 2. Significant underlying liver disease inhibiting curative liver resection according to preoperative imaging; 3 . Bilateral tumor extension to secondary biliary tree; 4 . Encasement or occlusion of the main portal vein; 5 . Lobar atrophy with contralateral portal vein or main hepatic artery involvement; 6. Contralateral tumor extension to secondary biliary tree; 7. Evidence of metastases to N2 level lymph nodes including peripancreatic (head only), paraduodenal, periportal, celiac, superior mesenteric, and/or posterior pancreaticoduodenal lymph nodes; 8 . Presence of distant metastases.

All following variables were recorded: demographic variables: age and gender; presence of pruritus: before and after MTP; laboratory tests: total bilirubin, aspartate aminotransferase (AST), alanine aminotransferase (ALT), and alkaline phosphatase (ALP) before and after MTP; survival time: OS - overall survival that is defined as survival after the time of diagnosis (month), CS - survival after catheter placement (month); interval time between diagnosis and catheter placement.

Imaging findings included: involvement of liver parenchyma, involvement of great vessels, regional lymph node invasion, tumor size, and staging: according to Bismuth-Corlette classification [12], based on the location and extent of the tumor.

Any related complications were also documented such as cholangitis: after MTP based on Charcot's cholangitis triad [13], gastrointestinal bleeding (GIB), and catheter infection based on catheter tip culture.

\section{Multimodality therapeutic protocol}

After percutaneous trans-hepatic cholangiography (PTC), an internal-external drainage catheter (F10-12) was placed in the appropriate location under fluoroscopy guidance. After drainage by internal-external biliary catheter, the patients underwent EBRT with high energy linear accelerator (6 or $18 \mathrm{MV}$ photon beams) to the clinical target volume (CTV), defined by primary tumor plus $2 \mathrm{~cm}$ margin around the tumor in addition to primary lymph nodes drainage including porta-hepatis, celiac, and pancreaticoduodenal lymph nodes. Planning target volume (PTV) defined as $1-2 \mathrm{~cm}$ margin around CTV, and prescribed dose for PTV was 45-50.4 Gy in 25-28 fractions. The patients underwent concurrent chemotherapy by capecitabine (Xeloda) $825 \mathrm{mg} / \mathrm{m}^{2} /$ bid at the days of radiotherapy. After completion of EBRT (after 7-10 days), in case of bilirubin $<5$ and normal liver function tests, the patients underwent emplacement of $120 \mathrm{~cm}$ brachytherapy catheter into internal-external catheter using fluoroscopy guidance, and after passing the catheter from luminal tumoral stricture, the brachytherapy catheter was fixed into the internal-external drainage catheter.

The patients underwent a multi-slice abdominal CT scan with $1 \mathrm{~mm}$ thickness before beginning of the treatment. CTV was defined as tumor volume in MRI (before EBRT), with $1-1.5 \mathrm{~cm}$ radially and $1-2 \mathrm{~cm}$ longitudinally around the catheter, in small number of patients with involvement of right and left hepatic duct at the same time, whose ducts were accessible by catheter, and both hepatic ducts were treated at the same time (Figure 1). The planning was done using a treatment planning software (Flexitron, ELEKTA ${ }^{\circledR}$ ), and after the planning and dosimetry, the patients were treated by brachytherapy machine (Flexitron, ELEKTA ${ }^{\circledR}$ ) with iridium-192 ( ${ }^{192}$ Ir) or cobalt-60 $\left({ }^{60} \mathrm{Co}\right)$ sources for $21 \mathrm{~Gy}$ in 3 fractions in 3 consecutive days (the prescribed dose to CTV was defined as $\mathrm{D}_{90}=3 \mathrm{~Gy}$ and $\mathrm{V}_{200}<20 \%$ ). A special attention was paid to the dose-volume histogram of planning with $200 \%$ of prescribed dose, which was limited only to the external surface of internal-external drainage catheter. The $\mathrm{EQD}_{2}(\alpha / \beta$ ratio $=10)$ of brachytherapy was $30 \mathrm{~Gy}$, and when adding the EBRT dose of 45-50 Gy, the total EQD 2 was 75-80 Gy.

After 4-6 weeks of BT completion, the catheter was removed. Patients who could not tolerate the internal-exter- 

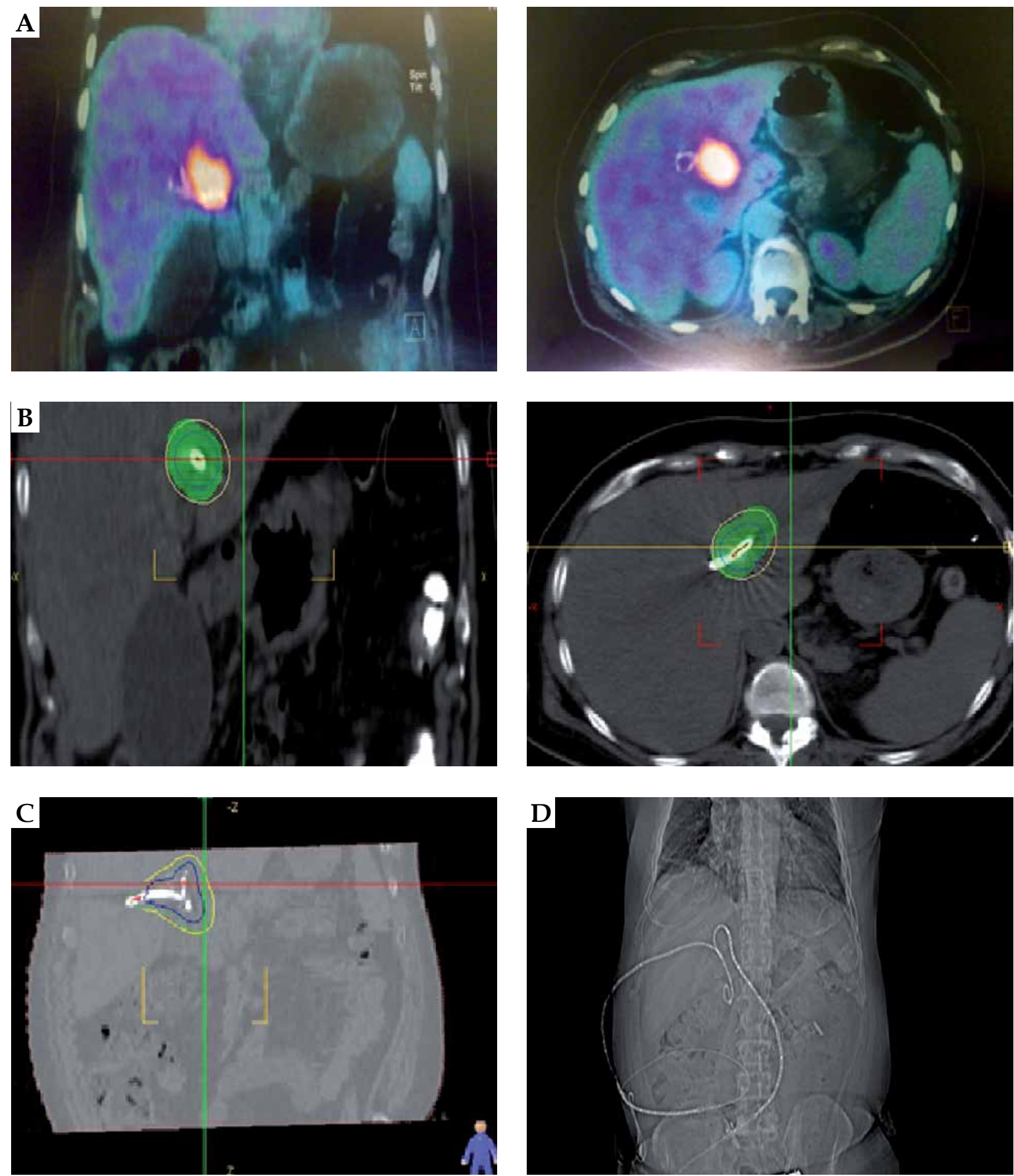

Fig. 1. A patient with cholangiocarcinoma involving both left and right hepatic ducts. A) Tumor uptake in positron emission tomography (PET) scan. B) Clinical target volume (CTV) and dose coverage in CT planning. C) CTV and dose coverage in CT planning. D) Bilateral catheter biliary drainage

nal catheter for a long time, BT performed before EBRT. In addition, three patients received induction chemotherapy with cisplatin and gemcitabine. There were no treatment breaks recorded in patients, no treatment-related toxicity in our patients that could cause treatment discontinuation, and the overall treatment time was 120 days in all patients. During follow-up, MRI/CT scan and lab data, including liver function test and tumor markers (i.e., CA19-9, CEA) were performed every three months.

\section{Ethics}

This study was conducted in accordance with the Declaration of Helsinki and approved by the ethics com- 
mittee of our institute. After the goals of the study were explained and the patients were assured that their individual data will remain confidential to the research team, all participants signed an informed consent.

\section{Statistical analysis}

Data analysis was performed using IBM SPSS 16 for Windows (Chicago, IL, USA), and a $p$-value $<0.05$ was considered statistically significant. Descriptive data (minimum, maximum, range, mean, and standard deviation) of all variables were calculated for all participants. Kolmogorov-Smirnov test was performed to determine the normality distribution of data. Wilcoxon test, a non-para- metric test, was applied to assess the association between variables with the abnormal distribution. Kaplan-Meier survival analysis was used to calculate survival. A logrank test was performed to compare the survival distributions of two samples.

\section{Results}

A total of 38 eligible patients, with mean SD age $=$ $58.08(9.80)$ years (range, $42-74)$, male $=22(57.9 \%)$, were evaluated. Mean SD of tumor size was 36.66 (12.94) mm (range, 17-70 mm). Regarding the tumor staging, $15(39.5 \%)$ were type IIIA, $5(13.2 \%)$ were type IIIB, $10(26.3 \%)$ were type IV, and $8(21.2 \%)$ were undefined.

Table 1. Prognostic factors affecting overall survival (OS) and survival after catheter placement (CS)

\begin{tabular}{|c|c|c|c|c|c|c|c|c|c|c|c|}
\hline \multirow[t]{3}{*}{ Variables } & \multirow[t]{3}{*}{$N(\%)$} & \multicolumn{5}{|c|}{ OS } & \multicolumn{5}{|c|}{ CS } \\
\hline & & \multirow[t]{2}{*}{ Mean } & \multirow[t]{2}{*}{ Median } & \multicolumn{2}{|c|}{$95 \% \mathrm{Cl}$ interval } & \multirow[t]{2}{*}{$P$-value } & \multirow[t]{2}{*}{ Mean } & \multirow[t]{2}{*}{ Median } & \multicolumn{2}{|c|}{$95 \% \mathrm{Cl}$ interval } & \multirow[t]{2}{*}{$P$-value } \\
\hline & & & & $\begin{array}{l}\text { Lower } \\
\text { limit }\end{array}$ & $\begin{array}{l}\text { Upper } \\
\text { limit }\end{array}$ & & & & $\begin{array}{l}\text { Lower } \\
\text { limit }\end{array}$ & $\begin{array}{l}\text { Upper } \\
\text { limit }\end{array}$ & \\
\hline \multicolumn{12}{|c|}{ Age groups } \\
\hline$<50$ & $10(26.3)$ & 11.90 & 8 & 8.11 & 15.63 & \multirow[t]{4}{*}{0.17} & 8.5 & 6 & 5.58 & 11.40 & \multirow[t]{4}{*}{0.07} \\
\hline $50-59$ & $9(23.6)$ & 17.33 & 15 & 14.26 & 20.40 & & 13.44 & 12 & 10.42 & 16.46 & \\
\hline $60-69$ & $14(35.8)$ & 14.07 & 15 & 10.97 & 17.16 & & 10.86 & 9 & 8.08 & 13.62 & \\
\hline $70-79$ & $5(13.1)$ & 20.40 & 20 & 16.83 & 23.96 & & 17.40 & 18 & 14.02 & 20.77 & \\
\hline \multicolumn{12}{|l|}{ Gender } \\
\hline Female & $16(42.1)$ & 13.06 & 13 & 10.63 & 15.49 & \multirow[t]{2}{*}{$0.03^{*}$} & 9.69 & 9 & 7.78 & 11.59 & \multirow[t]{2}{*}{$0.01^{\star}$} \\
\hline Male & $22(57.9)$ & 16.59 & 15 & 14.05 & 19.12 & & 13.18 & 10 & 10.80 & 15.55 & \\
\hline \multicolumn{12}{|c|}{$\begin{array}{l}\text { Liver parenchyma } \\
\text { involvement }\end{array}$} \\
\hline Yes & $21(55.3)$ & 12.10 & 12 & 9.92 & 14.26 & \multirow[t]{2}{*}{$<0.001^{\star}$} & 8.86 & 9 & 7.05 & 10.66 & \multirow[t]{2}{*}{$<0.001^{*}$} \\
\hline No & $17(44.7)$ & 18.82 & 17 & 16.32 & 21.41 & & 15.24 & 15 & 12.81 & 17.66 & \\
\hline \multicolumn{12}{|c|}{$\begin{array}{l}\text { Great vessels } \\
\text { involvement }\end{array}$} \\
\hline Yes & 15 (39.5) & 10.60 & 8 & 8.12 & 13.07 & \multirow[t]{2}{*}{$<0.001^{\star}$} & 7.53 & 6 & 5.76 & 9.30 & \multirow[t]{2}{*}{$<0.001^{*}$} \\
\hline No & $23(60.5)$ & 18.04 & 16 & 15.87 & 20.21 & & 14.43 & 14 & 12.33 & 16.53 & \\
\hline \multicolumn{12}{|c|}{ Bismuth stage } \\
\hline III & $20(52.6)$ & 17.74 & 16 & 14.09 & 20.01 & \multirow[t]{2}{*}{$<0.001^{*}$} & 14.40 & 14 & 11.97 & 16.82 & \multirow[t]{2}{*}{$<0.001$} \\
\hline IV & $10(26.3)$ & 7.50 & 6 & 5.90 & 9.09 & & 4.70 & 4 & 3.92 & 5.48 & \\
\hline \multicolumn{12}{|c|}{$\begin{array}{l}\text { Regional lymph } \\
\text { node involvement }\end{array}$} \\
\hline Yes & $21(55.3)$ & 12.76 & 10 & 10.15 & 15.36 & \multirow[t]{2}{*}{0.07} & 9.67 & 7 & 7.45 & 11.88 & \multirow[t]{2}{*}{$0.04^{*}$} \\
\hline No & $17(44.7)$ & 18.00 & 16 & 15.83 & 20.16 & & 14.24 & 13 & 12.08 & 16.38 & \\
\hline Tumor size & & & & & & & & & & & \\
\hline$<25$ & $5(12.8)$ & 19.80 & 20 & 17.23 & 22.36 & 0.29 & 17.00 & 18 & 13.74 & 20.25 & 0.07 \\
\hline$\geq 25$ & $33(87.2)$ & 14.39 & 15 & 12.38 & 16.40 & & 10.90 & 9 & 9.17 & 12.64 & \\
\hline
\end{tabular}

*statistically significant, OS - overall survival, CS - survival after catheter placement 
A

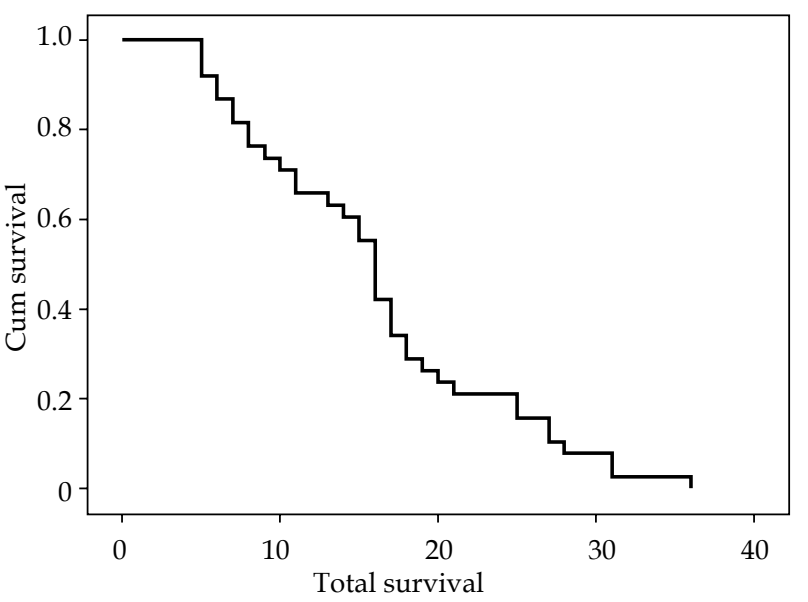

B

Survival function

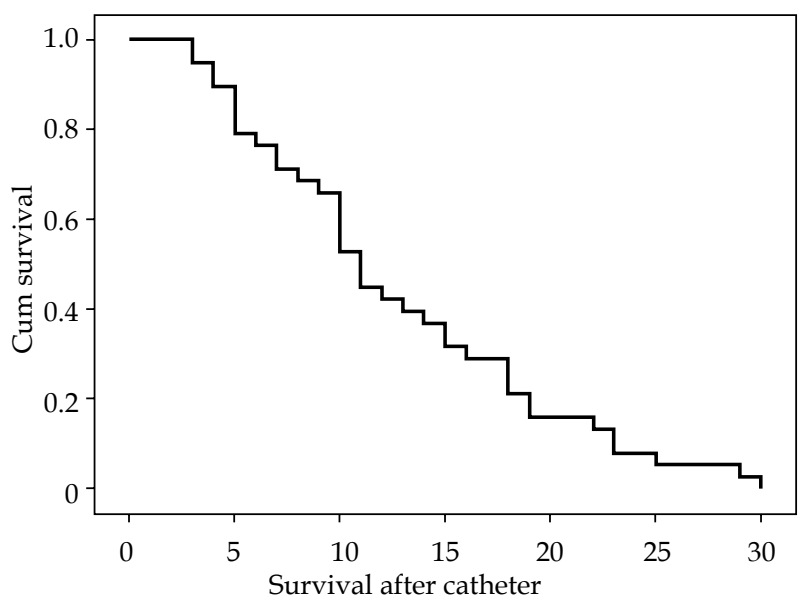

Fig. 2. Kaplan-Meier graph for (A) overall survival (OS), and (B) after catheter placement (CS) for all patients

Two of patients with metastasis were treated with brachytherapy and chemotherapy thereafter.

Of those, $21(55.3 \%), 15(39.5 \%)$, and $17(44.7 \%)$ presented with involvement of liver parenchyma, great vessels, and regional lymph nodes, respectively (Table 1). Catheter infection occurred in $5(13.2 \%)$ and cholangitis was detected in $2(5.3 \%)$ cases. GIB was also observed only in $1(2.6 \%)$ patient.

Mean SD of OS was 15.11 (8.10) months, with median $=15$ (95\% CI: 13.25-16.69) (Figure 2), and CS was 2-29 months, with mean SD = 11.71 (7.29) (median = 10; 95\% CI: 10.05-13.37) (Figure 2).

The interval time between diagnosis and catheter placement was 1-12 months, with mean SD = 3.39 (2.58). There was no significant relationship between the interval time between diagnosis and catheter placement in OS or CS ( $p=0.21$ and 0.07 , respectively).

Pruritus was reported in $35(92.1 \%)$ patients at the time of catheter placement and in follow-up, only $3(7.9 \%)$ cases of pruritus were reported. The difference in the number of patients with pruritus before and after catheter insertion was found to be significant $(p<0.001)$. However, no patient reported new-onset pruritus after catheter placement.

Our analysis showed a significant decline in serum levels of total bilirubin, AST, ALT, and ALP after catheter placement $(p<0.001$ for all variables) (Table 2$)$.

OS and CS did not indicate a significant difference between various age groups $(p=0.17$ and 0.07 , respec- tively) (Table 1). This association, however, was significant in terms of gender, where males were more likely to have higher OS (median $=15$ vs. 13 months) and CS (median $=10$ vs. 9 months) ( $p=0.03$ and 0.01 , respectively) (Table 1 and Figure 3).

Further analysis revealed a considerable decrease in OS and CS in patients with an involvement of liver parenchyma, great vessels, regional lymph nodes, and Bismuth type IV (Table 1). This correlation, however, was marginally lower for tumor $\geq 25 \mathrm{~mm}$ in size regarding CS $(p=0.07)$, but was not significant for OS $(p=0.29)$ (Table 1$)$.

\section{Discussion}

Treatment of choice in CCs, which show a higher survival rate (5-year survival of $10-40 \%$ ), is a surgical resection with a negative margin that often requires lymphadenectomy and major hepatectomy $[12,13]$. Unfortunately, only approximately $20 \%$ of patients are the candidates for radical resection, and the rest of patients benefit from palliative treatments [10]. Palliative options include surgical, percutaneous, and endoscopic palliations. The aim of palliative therapy is to relieve from obstructive symptoms (pain, jaundice, pruritus, and cholangitis) and quality of life improvement [14].

In patients with advanced cancers, percutaneous placement of the catheter is the first choice in palliative therapy, with low complications and mortality [10]. Moreover, radiotherapeutic modalities (EBRT and BT)

Table 2. Change of laboratory tests after catheter placement

\begin{tabular}{lccc}
\multirow{2}{*}{ Lab test } & \multicolumn{2}{c}{ Catheter placement } & $P$-value \\
\cline { 2 - 3 } & Before (mean (SD)) & After (mean (SD)) & $<0.001^{*}$ \\
\hline Total bilirubin & $11.88(4.12)$ & $3.35(2.07)$ & $<0.001^{*}$ \\
\hline AST & $127.42(117.69)$ & $84.69(89.54)$ & $<0.001^{*}$ \\
\hline ALT & $127.00(137.07)$ & $80.58(86.44)$ & $<0.001^{*}$
\end{tabular}

*statistically significant, AST - aspartate aminotransferase, ALT - alanine aminotransferase, ALP - alkaline phosphatase 
A

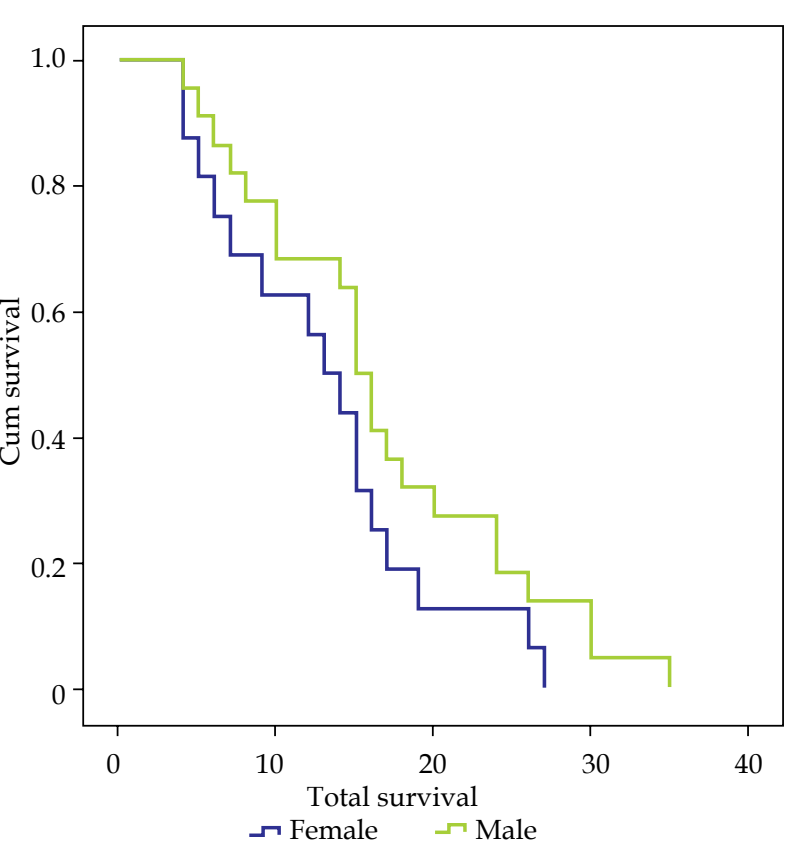

$\neg$ Female $\neg$ Male
B

Survival function

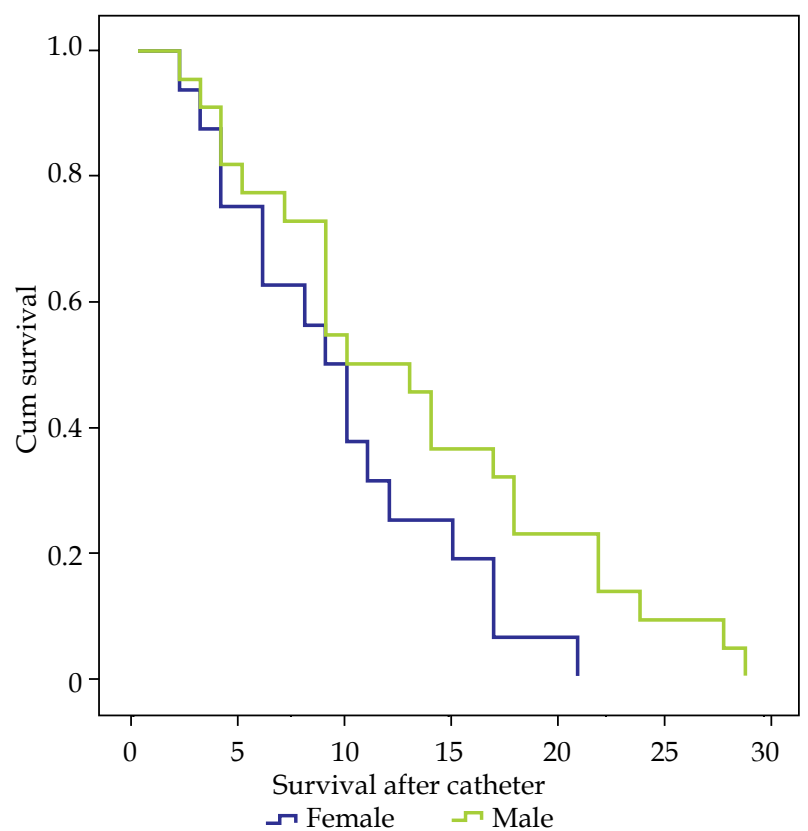

have been shown to have promising outcomes as a part of palliative therapy [15]. The addition of radiation to chemotherapy resulted in a significant improvement in 2 -year OS (25.8\% vs. $20.0 \%$ ) [16]. Another therapeutic approach is the combination of radiotherapy and chemotherapy to achieve a higher local and systemic control [17]. Hence, we defined the MTP as percutaneous intervention + EBRT + brachytherapy + systemic chemotherapy, to be administered in patients with unresectable ECCs.

The median survival after diagnosis (OS) and after catheter placement (CS) was measured as 15 and 10 months, respectively. Complications included: catheter infection, cholangitis, and GIB reported in $13.2 \%, 5.3 \%$, and $2.6 \%$, respectively. Our total outcomes (survival time and complication rates) were comparable to the following previous studies. Earlier studies, with an almost similar protocol, reported the same survival rates $[15,16,17]$. Other papers, however, showed higher survival time, but complications were significantly higher, comparing to the current study $[18,19]$. There are several studies that did not use systemic chemotherapy, and their results showed a lower survival time $[20,21,22,23]$.

In a study by Mahadevan et al. [18], patients were treated with stereotactic body radiotherapy (SBRT) at a median dose of 30 Gy daily in three fractions, along with chemotherapy with gemcitabine, and reported a 17-month survival at the end of study. They reported complications as a duodenal ulcer $(5.8 \%)$, cholangitis $(2.9 \%)$, and liver abscess $(2.9 \%)$. An increased survival rate and lower total complications have been reported, as compared with our study.

Barney et al. [24] used SBRT using a dose of 45-60 Gy every 3 to 5 days, over one week, with a median prescription dose of $55 \mathrm{~Gy}$, and chemotherapy was used before radiotherapy. They eventually reported one-year survival for their patients, in which a case of hepatic failure
(10\%) and a case of biliary obstruction (10\%) were reported. This study has resulted in less survival and more complications, as compared with the current study.

In another study by Foo et al. [19], patients underwent EBTR, BT, and chemotherapy, and a median survival of 12.8 months was reported. In a previous study by Takamura et al. [15], patients underwent EBRT, BT, and stent insertion, with a median survival rate of oneyear. Furthermore, Shinohara et al. [25] treated patients with EBRT and BT without using chemotherapy, and described an 11-month survival at the end of study.

Autorino and colleagues [26] presented 27 patients with unresectable ECC. One group of patients underwent EBRT + concurrent gemcitabine, and the other group was treated with EBRT+ concurrent gemcitabine + intraluminal HDR brachytherapy with 15-20 Gy. Median OS and LC were significantly better in the brachytherapy group.

This discrepancy in survival time among various studies might be due to the following reasons: 1. Patients' inequality in demographic features and comorbidities status; 2 . Difference in staging system (Bismuth vs. the American Joint Committee on Cancer - AJCC); 3. Heterogeneity in patients regarding their tumor stage; 4 . Dissimilarity in defining and techniques of performing the MTP in terms of EBRT and brachytherapy dosages, dosage and type of the drug combination in chemotherapy, and period of administration.

Furthermore, the current study revealed a negative impact of the involvement of liver parenchyma, great vessels, regional lymph nodes, and higher tumor staging according to Bismuth classification on survival time in patients who underwent the MTP. No considerable difference, however, was observed in different groups regarding age and tumor size; however, the survival time was significantly higher in males. 
Similar prognostic factors have been reported in lymph node metastasis [27] and tumor stage [28]. In Wellner's study [27], adjuvant chemotherapy after surgery did not play a role in patients with prognosis. However, results in terms of perineural involvement, lymph node metastasis, positive surgical margin, and undifferentiated adenocarcinoma were found to be associated with survival. Another study by Vern-Gross [29] also suggested that local involvement and distant metastasis were effective factors in survival, while additional radiotherapy for post-surgical treatment influenced the improvement of overall survival or cancer-specific survival (CSS) in patients. Fuller et al. [28] reported the following factors to be effective in the survival of patients with ECCs including age, AJCC stages, tumor grade, and usage of surgery or radiotherapy. Murakami et al. [30] also considered adjuvant chemotherapy, positive surgical margin, and lymph node metastases as significant factors in patients' survival.

In addition to the variety of therapeutic approaches, the difference in reported prognostic factors can be due to the varying number of patients in each study and the duration of patients' follow-up.

Limited sample size and lack of control group were the most substantial limitation of the present study. Therefore, further investigations, particularly randomized clinical trials with a large sample size, regarding the efficacy of different chemotherapy regimens and combination with EBRT and brachytherapy, are recommended.

\section{Conclusions}

The multimodality therapeutic approach in patients with inoperable ECCs could definitely improve their survival time and decrease complications. Survival time is significantly depended on tumor staging, gender, and involvement of liver parenchyma, great vessels, and regional lymph nodes.

\section{Acknowledgments}

The authors are thankful to all participated patients and hospital staff for their collaboration.

\section{Disclosure}

The authors report no conflict of interest.

\section{References}

1. Khan SA, Thomas HC, Davidson BR et al. Cholangiocarcinoma. Lancet 2005; 366: 1303-1314.

2. Banales JM, Cardinale V, Carpino G et al. Cholangiocarcinoma: current knowledge and future perspectives consensus statement from the European Network for the Study of Cholangiocarcinoma (ENS-CCA). Nat Rev Gastroenterol Hepatol 2016; 13: 261-280.

3. Shaib Y, El-Serag H. The epidemiology of cholangiocarcinoma. Semin Liver Dis 2004; 24: 115-125.

4. Jung DH, Kim M-S, Cho CK et al. Outcomes of stereotactic body radiotherapy for unresectable primary or recurrent cholangiocarcinoma. Radiat Oncol J 2014; 32: 163-169.

5. Yee K, Sheppard BC, Domreis J et al. Cancers of the gallbladder and biliary ducts. Oncology (Williston Park) 2002; 16: 939946,949 .
6. Cardinale V, Bragazzi MC, Carpino G et al. Cholangiocarcinoma: increasing burden of classifications. Hepatobiliary Surg Nutr 2013; 2: 272-280.

7. Qureshi K, Jesudoss R, Al-Osaimi AMS. The treatment of cholangiocarcinoma: a hepatologist's perspective. Curr Gastroenterol Rep 2014; 16: 412.

8. Cho MS, Kim SH, Park SW et al. Surgical outcomes and predicting factors of curative resection in patients with hilar cholangiocarcinoma: 10-year single-institution experience. J Gastrointest Surg 2012; 16: 1672-1679.

9. Ito F, Cho CS, Rikkers LF et al. Hilar cholangiocarcinoma: current management. Ann Surg 2009; 250: 210-218.

10. Golfieri R, Giampalma E, Renzulli M et al. Unresectable hilar cholangiocarcinoma: multimodality approach with percutaneous treatment associated with radiotherapy and chemotherapy. In Vivo 2006; 20: 757-760.

11. Chamberlain RS, Blumgart LH. Hilar cholangiocarcinoma: a review and commentary. Ann Surg Oncol 2000; 7: 55-66.

12. Bismuth $H$, Nakache R, Diamond T. Management strategies in resection for hilar cholangiocarcinoma. Ann Surg 1992; 215: 31-38.

13. Rumsey S, Winders J, MacCormick AD. Diagnostic accuracy of Charcot's triad: a systematic review. ANZ J Surg 2017; 87: 232-238.

14. DeOliveira ML, Cunningham SC, Cameron JL et al. Cholangiocarcinoma: thirty-one-year experience with 564 patients at a single institution. Ann Surg 2007; 245: 755-762.

15. Takamura A, Saito H, Kamada $\mathrm{T}$ et al. Intraluminal lowdose-rate 192Ir brachytherapy combined with external beam radiotherapy and biliary stenting for unresectable extrahepatic bile duct carcinoma. Int J Radiat Oncol Biol Phys 2003; 57: 1357-1365.

16. Jackson MW, Amini A, Jones BL et al. Treatment selection and survival outcomes with and without radiation for unresectable, localized intrahepatic cholangiocarcinoma. Cancer J 2016; 22: 237-242.

17. Morganti AG, Trodella L, Valentini V et al. Combined modality treatment in unresectable extrahepatic biliary carcinoma. Int J Radiat Oncol Biol Phys 2000; 46: 913-919.

18. Mahadevan A, Dagoglu N, Mancias J et al. Stereotactic body radiotherapy (SBRT) for intrahepatic and hilar cholangiocarcinoma. J Cancer 2015; 6: 1099-1104.

19. Foo ML, Gunderson LL, Bender CE et al. External radiation therapy and transcatheter iridium in the treatment of extrahepatic bile duct carcinoma. Int J Radiat Oncol Biol Phys 1997; 39: 929-935.

20. Cho MS, Kim SH, Park SW et al. Surgical outcomes and predicting factors of curative resection in patients with hilar cholangiocarcinoma: 10-year single-institution experience. J Gastrointest Surg 2012; 16: 1672-1679.

21. Minsky BD, Kemeny N, Armstrong JG et al. Extrahepatic biliary system cancer: an update of a combined modality approach. Am J Clin Oncol 1991; 14: 433-437.

22. Momm F, Schubert E, Henne K et al. Stereotactic fractionated radiotherapy for Klatskin tumours. Radiother Oncol 2010; 95: 99-102.

23. Polistina FA, Guglielmi R, Baiocchi $\mathrm{C}$ et al. Chemoradiation treatment with gemcitabine plus stereotactic body radiotherapy for unresectable, non-metastatic, locally advanced hilar cholangiocarcinoma. Results of a five year experience. Radiother Oncol 2011; 99: 120-123.

24. Barney BM, Olivier KR, Miller RC et al. Clinical outcomes and toxicity using Stereotactic Body Radiotherapy (SBRT) for advanced cholangiocarcinoma. Radiat Oncol 2012; 7: 67.

25. Shinohara ET, Guo M, Mitra N et al. Brachytherapy in the treatment of cholangiocarcinoma. Int J Radiat Oncol 2010; 78: 722-728. 
26. Autorino R, Mattiucci GC, Ardito F et al. Radiochemotherapy with gemcitabine in unresectable extrahepatic cholangiocarcinoma: long-term results of a phase II study. Anticancer Res 2016; 36: 737-740.

27. Wellner UF, Shen $Y$, Keck T et al. The survival outcome and prognostic factors for distal cholangiocarcinoma following surgical resection: a meta-analysis for the 5-year survival. Surg Today 2017; 47: 271-279.

28. Fuller CD, Wang SJ, Choi M et al. Multimodality therapy for locoregional extrahepatic cholangiocarcinoma: a population-based analysis. Cancer 2009; 115: 5175-5183.

29. Vern-Gross TZ, Shivnani AT, Chen K et al. Survival outcomes in resected extrahepatic cholangiocarcinoma: effect of adjuvant radiotherapy in a surveillance, epidemiology, and end results analysis. Int J Radiat Oncol 2011; 81: 189-198.

30. Murakami Y, Uemura K, Sudo T et al. Prognostic factors after surgical resection for intrahepatic, hilar, and distal cholangiocarcinoma. Ann Surg Oncol 2011; 18: 651-658. 